\title{
Beratung für die »Generation 65+«
}

\author{
Ute Acker-Wild
}

Entsprechend der demografischen Entwicklung hat die Zahl der älteren psychisch kranken Menschen zugenommen und wird weiterhin ansteigen. $\mathrm{Zu}$ den langjährigen - zum Teil chronischen - psychischen Erkrankungen der ambulant betreuten Personen kommt zwangsläufig der individuelle Abbauprozess hinzu. Neben der psychiatrischen psychosozialen Betreuung entstehen zunehmend Pflege- und andere Versorgungsbedarfe. Ein Projekt in Frankfurt am Main sucht nach praktischen Möglichkeiten der Durchsetzung neuer Hilfen.

Ausgenommen die altgewordenen psychisch kranken Menschen, die bereits lange Jahre in einer psychiatrischen Versorgungsstruktur eingebunden sind, gibt es keine fundierten Kenntnisse über so genannte Risikogruppen wie beispielsweise sozial isoliert oder benachteiligte, psychisch veränderte, und psychosozial auffällige Menschen. Auch in klassischen Arbeitsfeldern der Altenhilfe ist der Anteil an Menschen mit einer psychiatrischen Erkrankung oder einer psychiatrischen Auffälligkeit bisher nicht erhoben. Nach Auskunft der Fachkräfte zeigen in Altenwohnanlagen immer mehr Bewohnerinnen und Bewohner Symptome einer psychischen Erkrankung. Klubleiterinnen berichten über Besucher und Besucherinnen mit psychiatrischen Auffälligkeiten. Nicht zuletzt leben in Alten- und Pflegeheimen psychisch kranke Menschen, die bei einer adäquaten ambulanten Versorgung in einer eigenen Wohnung hätten bleiben können.

In einem zweijährigen Projekt zur Situation psychisch kranker alter Menschen in Frankfurt am Main haben Mitarbeitende der Psychosozialen Dienste des Frankfurter Vereins für soziale Heimstätten Grundlagenmaterial zur Entwicklung eines speziellen Angebotes erarbeitet und ein Konzept erstellt. Dieses berücksichtigt die spezifische Altersproblematik des Personenkreises und den Mehrbedarf an Pflege und psychosozialer Betreuung. Eingebunden ist das Konzept in vorhandene Strukturen der Psychosozialen Dienste des Frankfurter Vereins für soziale Heimstätten (vgl. Abb. 1.)

\section{Das Projekt}

Ziele des Projektes »Betreuungsbedarf für alt gewordene psychisch kranke Menschen im Betreuten Wohnen« sind:

- Klärung des Betreuungsbedarfs für psychisch kranke alte Menschen - quantitativ und qualitativ

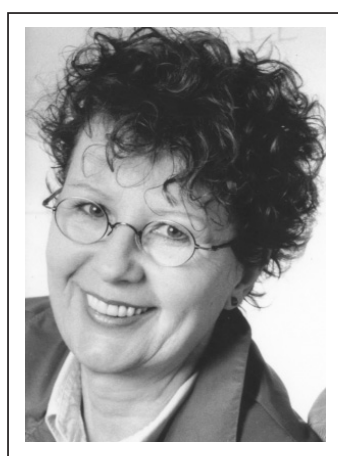

Ute Acker-Wild ist DiplomSozialarbeiterin und seit 17 Jahren Mitarbeiterin im Betreuten Wohnen und in einer Psychosozialen Kontakt- und Beratungsstelle des Frankfurter Vereins für soziale Heimstätten e. V. Sie leitet zusätzlich das Projekt »Ambulante Betreuung für altgewordene psychisch kranke alte Menschen «; weitere Projektmitarbeitende sind Elvira Becker-Schmidt, Matthias Gerstel, Kai Marschner und Karin Riemann. E-Mail psd.b@frankfurter-verein.de

- Entwicklung eines Konzeptes zur Weiterentwicklung des Angebots im Betreuten Wohnen und in der Psychosozialen Kontakt- und Beratungsstelle für altgewordene psychisch kranke Menschen über 65 Jahre

- Implementierung und Erprobung des Konzeptes

- Entwicklung von Standards zur Betreuung des Personenkreises

- Modifizierung der entsprechenden Instrumente zur Qualitätssicherung

- Ergänzung der Nutzerbefragung um den Aspekt »Betreuung altgewordener psychisch kranker Menschen im Betreuten Wohnen«

Im Folgenden werden die Aspekte der Projekterarbeitung dargestellt, die maßgeblich für Erarbeitung des Konzeptes waren. Ziel der verschiedenen Datenerhebungen war, den Betreuungsbedarf für Menschen über 65 Jahre im Frankfurter Westen zu erfassen. Aus den Ergebnissen lassen sich Tendenzen für eine zielgerichtete Weiterentwicklung der ambulanten Versorgung psychisch kranker alter Menschen ableiten.

In den ambulanten, stationären und teilstationären Diensten und Einrichtungen des Frankfurter Vereins für soziale Heimstätten - Betreutes Wohnen, Reha-Werkstätten, Tagesstätten, Begegnungsstätten, Meta-Quark-Haus (Wohnheim für psychisch kranke Menschen) - wurde eine Erhebung durchgeführt. Hier wurden Fragen zur Altersstruktur, zur Wohnsituation, der familiären Situation 


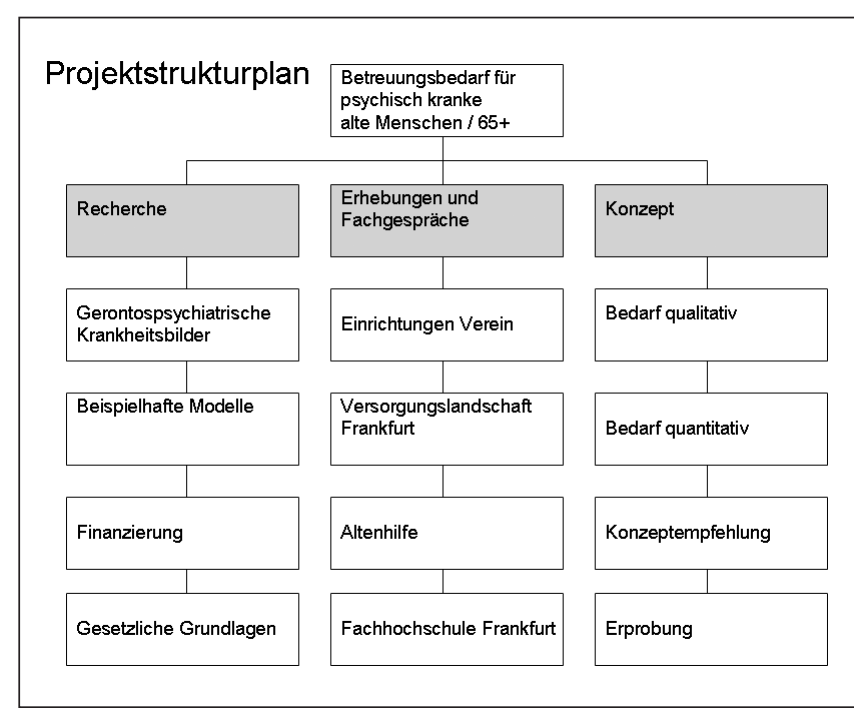

Abb. 1: Der Projektstrukturplan zeigt die Vorgehensweise des Frankfurter Vereins für soziale Heimstätten e. V.

von 438 Personen in den 3 Alten- und Pflegeheimen in Frankfurt-West haben ...

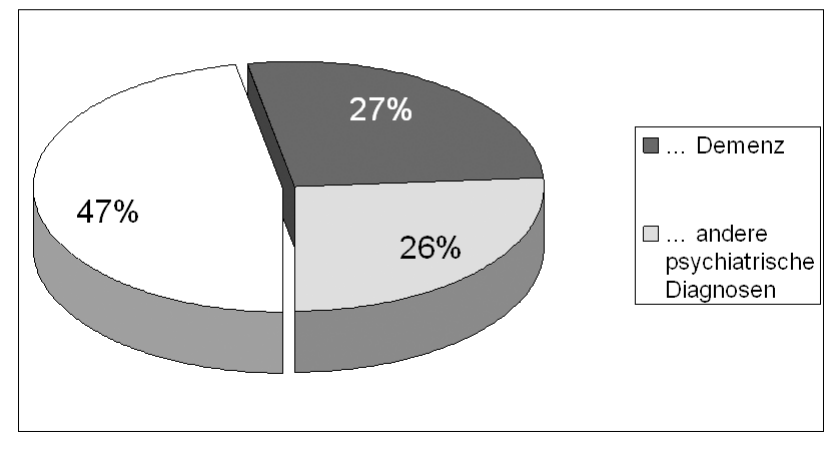

Abb. 2: Nach einer Befragung von drei großen Alten- und Pflegeheimen in Frankfurt am Main-West haben von 438 Personen mehr als die Hälfte eine Demenz oder eine andere psychiatrische Erkrankung.

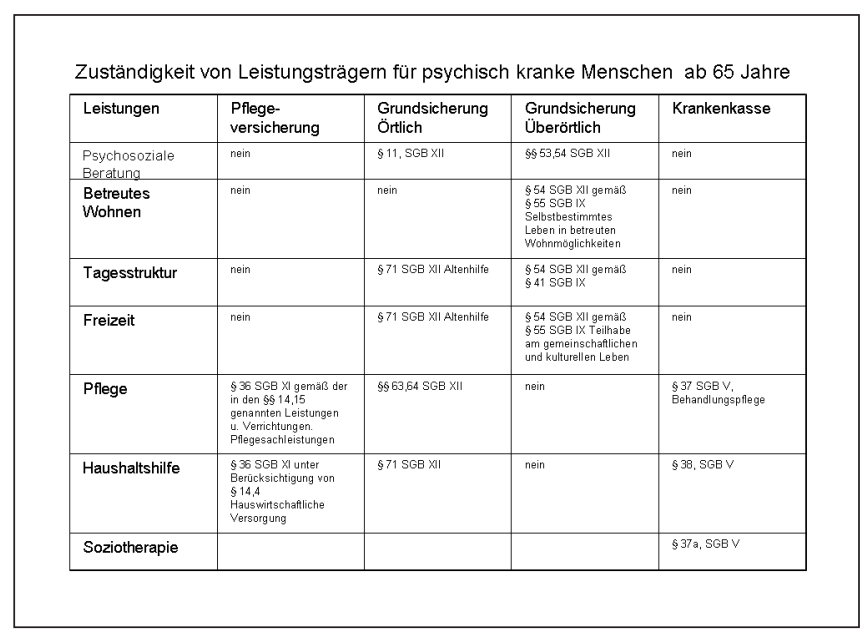

Abb. 3: Bei psychisch kranken Menschen ab 65 Jahren sind wenn überhaupt - unterschiedliche Leistungsträger zuständig. und die Anbindung an eine gesetzliche Betreuung gestellt. Die Ergebnisse zur Altersstruktur entsprechen im wesentlichen der demografischen Entwicklung. Einige wichtige Aspekte:

- Ein Viertel der betreuten Personen in diesen Einrichtungen ist über 55 Jahre alt.

- Der Frauenanteil ist bei den Älteren generell höher.

- 28 Prozent aller Betreuten haben einen gesetzlichen Betreuer.

- Im Betreuten Wohnen sind bereits bei 30 Prozent der betreuten Personen zusätzlich ambulante Dienste integriert.

\section{Erhebungen in Alten- und Pflegeheimen}

Ziel der Befragung war es, Erkenntnisse zu gewinnen über: Belegung, psychiatrische Erkrankungen, Versorgungssituation in der Einrichtung, Facheinschätzung zur Versorgung psychisch kranker Menschen in Alten- und Pflegeheimen. Die Befragung wurde in den drei größten Alten- und Pflegeeinrichtungen im Frankfurter Westen durchgeführt.

Zur Facheinschätzung der Pflegekräfte in Alten- und Pflegeheimen: Bezüglich der Betreuung von Menschen mit einer psychiatrischen Erkrankung wird ein spezieller Bedarf im Hinblick auf die Einzelbetreuung und die psychosoziale Beratung in Alten- und Pflegeheimen gesehen (vgl. Abb. 2). Für diesen speziellen Versorgungsbedarf gibt es keine personellen Ressourcen in der Einrichtung. Nur wenig Fachpersonal verfügt über eine gerontopsychiatrische Zusatzausbildung. Zirka zehn Prozent der in Altenund Pflegeheimen lebenden Bewohnerinnen und Bewohner könnten mit einem entsprechenden Unterstützungsangebot noch in einer eigenen Wohnung leben.

\section{Finanzierung}

Einen wesentlichen Teil der Überlegungen für ein Betreuungsangebot für Menschen über 65 Jahre nahmen die Überlegungen zur Finanzierung ein. Zwar gibt es für fast alle erforderlichen Leistungen Finanzierungsmöglichkeiten auf der jeweiligen gesetzlichen Grundlage (vgl. Abb. 3). Jedoch wird es verwaltungstechnisch sehr aufwendig sein, die jeweiligen Leistungen bei den entsprechenden Stellen zu beantragen. Eine Empfehlung lautet deshalb, zweckgebundene Kooperationen innerhalb der Fachbereiche zu entwickeln und Finanzierungsmodelle zu entwickeln, die eine Vereinfachung bringen.

\section{Das Konzept "Gerontopsychiatrische Beratung und Betreuung 65+«}

Aus den Erkenntnissen der umfangreichen Projektarbeit, zahlreichen Gesprächen mit Fachkollegen und Fachkolleginnen aus den Bereichen Psychiatrie und Altenhilfe 


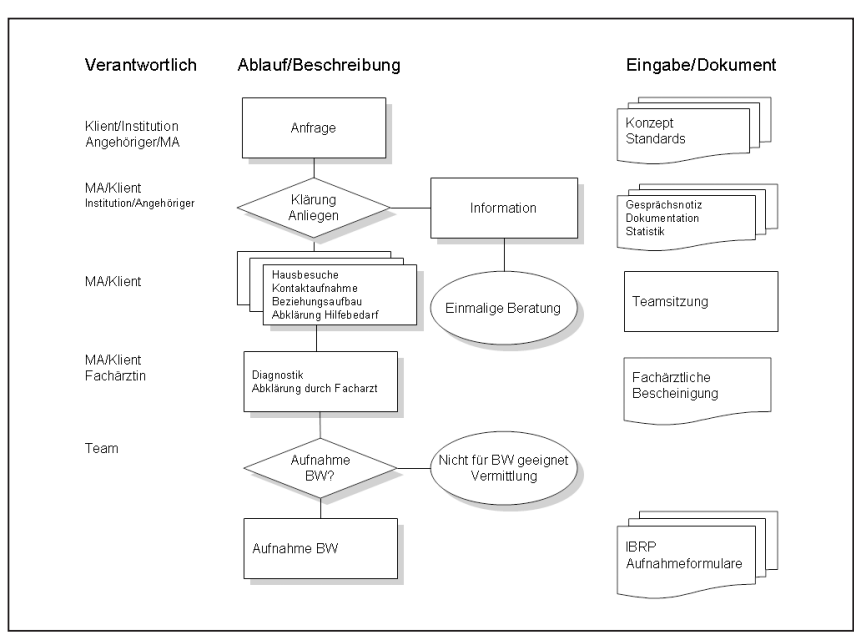

Abb. 4: Eine individuelle Hilfeplanung mit der betreuten Person und ihren Angehörigen steht am Anfang der Zusammenarbeit.

\section{Eigenständig leben mit psychischer Erkrankung - zwei Beispiele}

- Frau X, 78 Jahre alt, lebt in einer Zweizimmerwohnung im Stadtteil Sossenheim in Frankfurt am Main. Die Tür lässt sich kaum öffnen, da der Fußboden übersät ist mit Unrat. Um sich hinsetzen zu können, muss man bergeweise die Wäsche vom Stuhl räumen. Essensreste liegen umher. Der Kühlschrank ist schon lange nicht mehr im Betrieb. Frau X läuft geschäftig umher, redet unablässig von der Weltverschwörung und schimpft über ihren Bruder, der sie einst um das gemeinsame Erbe gebracht habe. Man muss die Stimme senken und leise reden, da die Wohnung komplett mit Mikrofonen ausgestattet sei und jedes Wort vom CIA mitgehört werde. Dies sei schon lange so und werde sich auch nicht mehr ändern. Frau X hat sich damit arrangiert und ihr Leben entsprechend darauf eingestellt. Den Sozialarbeiter, der sie freundlich darauf hinweist, dass es sinnvoll sein könnte, Unterstützung in Anspruch zu nehmen, lächelt sie an und lehnt dankend ab. Hilfe brauche sie nun wirklich nicht.

- Frau Y, 71 Jahre alt, lebt in einer Einhalbzimmerwohnung im 14. Stockwerkes eines Hochhauses in Frankfurt am Main-Niederrad. Die Wohnung ist geschmackvoll eingerichtet und in einem akkuraten Zustand. Frau Ys Unterarme sind verbunden, da Sie sich blutig gekratzt hat. Sie fragt höflich, ob sie in ihrer eigenen Wohnung rauchen darf. Danach raucht sie Kette. Vom äußeren Erscheinungsbild wirkt sie wie 85 , sie ist nahezu auf die Knochen abgemagert. Ihre Stimme ist leise und kaum vernehmlich. Sie erzählt aus einer bewegten Vergangenheit mit vielen Stationen, die sie in ihrem Leben gemacht hat. Jetzt kommt sie alleine nicht mehr aus dem Haus und braucht Unterstützung bei fast allen Verrichtungen. Frau Y ist dankbar für den Besuch des Sozialarbeiters. Vielleicht kann sie mit der Unterstützung »Betreutes Wohnen « nun doch weiterhin in der geliebten eigenen Wohnung bleiben. und den gesetzlichen Rahmenbedingungen haben wir ein Konzept für die ambulante Betreuung altgewordener psychisch kranker Menschen entwickelt. Dieses berücksichtigt die spezifische Altersproblematik des Personenkreises und den Mehrbedarf an Pflege und psychosozialer Betreuung. Eingebunden ist das Konzept in vorhandene Strukturen der ambulanten psychosozialen Versorgung.

\section{Zielgruppe}

Das ambulante Angebot richtet sich an Menschen ab dem 65 . Lebensjahr (»65+«), bei denen eine psychische Erkrankung oder seelische Behinderung vorliegt oder die davon bedroht sind. In dieser Altersphase zeigen sich oft komplexe multimorbide Einschränkungen, die zu berücksichtigen sind. Menschen mit einer eindeutigen demenziellen Erkrankung kann das Angebot übrigens nicht berücksichtigen, da die konzeptionelle Ausrichtung eine andere ist und zudem für an Demenz erkrankte Menschen vermehrt Versorgungsangebote entwickelt werden.

Folgende Personen sollen erreicht werden:

- alt gewordene psychisch kranke Menschen, die bereits in psychosoziale Hilfen eingebunden waren, jedoch altersbedingt aus der Hilfegewährung »herausfallen« (z.B. Reha-Werkstatt-Besucher und wegen Erreichen der Altersgrenze),

- alt gewordene psychisch kranke Menschen, die ein altersangepasstes Unterstützungsangebot benötigen

- Menschen, die im Alter erstmals psychisch erkranken oder auffällig werden

- psychisch kranke alte Menschen, bei denen unter Inanspruchnahme ambulanter Hilfen eine vorschnelle Heimeinweisung (z. B. nach einem Klinikaufenthalt) abgewendet und die eigenständige Wohnsituation erhalten werden kann.

Das Betreuungsangebot berücksichtigt zum einen die relevanten sozialpsychiatrischen Aspekte, wie sie im Betreuten Wohnen entwickelt wurden. Darüber hinaus sind Grundlagen der Betreuung Erkenntnisse aus der geriatrischen Alternsforschung. Diese beziehen sich auf das »Kompetenzmodell « (Baltes, Kruse, Lehr, Obrich, Thomae). Das Modell richtet den Blick auf das, was ein alter Mensch noch kann und bezieht sich vor allem auf Selbstbestimmung und Eigenverantwortung. Zusammenfassend verstehen wir unter Kompetenz die Fähigkeiten und Fertigkeiten einer Person, die zur Aufrechterhaltung eines selbstständigen, sinnerfüllten Lebens notwendig ist. Erforderlich sind die Aktivierung verschütteter Fähigkeiten, Stärkung vorhandener Kompetenzen und die Entwicklung neuer Perspektiven.

Da psychisch kranke alte Menschen häufig Schwierigkeiten haben, einen Hilfebedarf zu formulieren und Hilfen in Anspruch zu nehmen, soll der Erstkontakt möglichst niedrigschwellig erfolgen. Deshalb gehören Hausbesuche und aufsuchende Kontakte zu Regelleistungen. Eine solide Basisarbeit und ein vertrauensvoller Be- 
ziehungsaufbau sind wichtige Voraussetzungen, damit weitere Hilfen angenommen werden können. In Zusammenarbeit mit Klientin oder Klient und gegebenenfalls mit Angehörigen und gesetzlicher Betreuung erfolgt eine individuelle Hilfeplanung (vgl. Abb. 4).

\section{Erfahrungen}

Seit gut einem Jahr arbeiten wir nach dem Konzept »Gerontopsychiatrische Beratung und Betreuung 65+«. Neben den langjährig von uns bereuten altgewordenen Klientinnen und Klienten, für die wir jeweils das Betreuungsangebot ergänzen, gibt es erste Anfragen zur Betreuung von Personen über 65 Jahre, die erstmals Betreuung aus dem psychiatrischen Kontext anfragen. Der jeweilige Zugang ist entweder über die gesetzliche Betreuung, aus dem Bereich »Ambulante Psychiatrische Akutbehandlung zu Hause « des »Bamberger Hofes « in Frankfurt am Main oder Anfragen von Angehörigen. Wir stellen fest:

- Das Clearing mit der Kontaktaufnahme und der Abstimmung des Hilfebedarfs ist viel zeitaufwendiger als im klassischen Betreuten Wohnen. Das bedeutet: Diese Arbeit muss derzeit durch die Psychosoziale Kontakt und Beratungsstelle geleistet werden.

- Die Schnittstellen zur Altenhilfe sind (noch) unklar mit der Gefahr, dass unsere Arbeit eher als Konkurrenz denn als fachliche Unterstützung angesehen wird.

- Die Personalausstattung im Betreuten Wohnen ist völlig unzureichend, da der Arbeitsaufwand durch die Finanzierung der Dienste durch Fachleistungsstunden er- heblich gestiegen ist und die Betreuung für Personen über 65 Jahren zusätzlich zum bisherigen Platzkontingent geleistet werden muss (keine Finanzierung durch den Landeswohlfahrtsverband Hessen).

- Die Hilfebedarffeststellung mittels Integrierten Behandlungs- und Rehabilitationsplan (IBRP) ist ungeeignet, da durch die sehr ausführliche und differenzierte Beschreibungen die zu betreuenden Personen überfordert werden.

Aus den Erfahrungen sind die nächsten Handlungsschritte abzuleiten. So müssen jetzt auf der Ebene der Steuerung effektive Formen der Finanzierung entwickelt und Schnittstellen zwischen den Bereichen der Altenhilfe geklärt werden. Es gilt, neue Kooperationsformen zwischen Altenhilfe und Psychiatrie zu entwickeln, damit die vorhandenen Angebote beider Bereiche optimal genutzt werden können. Bezüglich der Bedarfsfeststellung und der Hilfeplanung ist es erforderlich, geeignete Instrumente zu entwickeln oder bereits vorhandene - gegebenenfalls geeignetere - zu modifizieren.

Wie hoch der tatsächliche Bedarf an Betreuung für diesen Personenkreis ist, können wir nur spekulieren, da es, wie bereits ausgeführt, keine gesicherten Daten gibt. Wir waren mit der Veröffentlichung des Angebotes bisher zögerlich, da wir die Betreuung zusätzlicher Personen mit der derzeitigen personellen Ausstattung nicht leisten können. Wenn die entsprechenden Bedingungen geschaffen sind, könnte eine Information über das Angebot bei Hausärzten, Sozialbezirksvorsteherinnen, Polizei, gesetzlichen Betreuungsstellen, Einrichtungen der Altenhilfe, den Sozialrathäuser, und den Pflegedienste nützlich sein.

\title{
Der „Klassiker“ - noch immer konkurrenzlos.
}

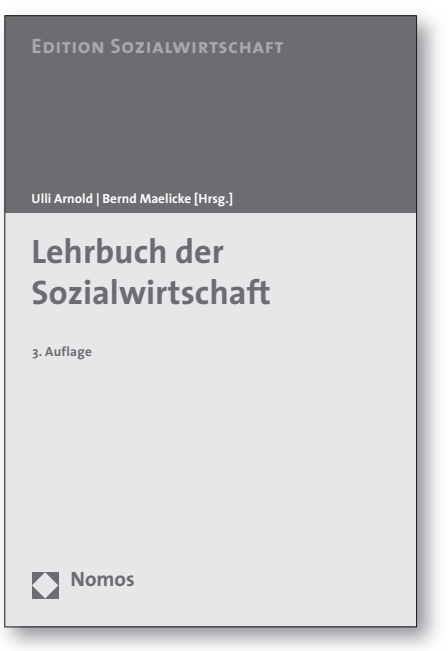

\author{
Lehrbuch für Sozialwirtschaft \\ Von Prof. Dr. Dr. h.c. Ulli Arnold, Universität Stuttgart und \\ Prof. Dr. Bernd Maelicke, Universität Lüneburg \\ 3. Auflage 2007, ca. 600 S., brosch., ca. 39,- €, ISBN 978-3-8329-2680-9 \\ (Edition Sozialwirtschaft, Bd. 9) \\ Erscheint Dezember 2007
}

Die vollständig aktualisierte und neubearbeitete 3 . Auflage vermittelt Basiswissen und aktuelle Informationen zu den wichtigsten Themen wie z. B.

- Entwicklung der sozialpolitischen Rahmenbedingungen

- Anbieter, Wettbewerber, Handlungsfelder, Dienstleistungen

- Typologie und Rechtsformen sozialwirtschaftlicher Organisationen

- Qualitätsmanagement, Sozialmarketing

- Finanzierungsmanagement, Fundraising

- Grundlagen des Managements in der Sozialwirtschaft 